probably involves inappropriate antigen presentation.

The authors suggest that the TBP amino-terminus downregulates antigen presentation by MHC class I. They speculate that the amino-terminus might have co-evolved with the MHC system to regulate its expression. In mice, the placenta is simply the first vital location of this function. It will be interesting to determine whether mutant adults have defects in other MHC class-I-dependent responses, such as susceptibility to intracellular pathogens.

Kirsty Minton

(D) References and links ORIGINAL RESEARCH PAPER Hobbs, N. K. et al. Removing the vertebrate-specific TBP $\mathrm{N}$-terminus disrupts placental $\beta_{2} \mathrm{~m}$-dependent interactions with the maternal immune system. Cell 110, 43-54 (2002) FURTHER READING Erlebacher, A. Why isn't the fetus rejected? Curr. Opin. Immunol. 13, 590-593 (2001)

tyrosine phosphorylation of $\mathrm{CD} 3 \zeta$ and other substrates is detected. The common belief that ITAM phosphorylation is the earliest event in TCR signalling needs rethinking. The early association of NCK with TCR-CD3 is physiologically relevant, because inhibition of this interaction decreases the number of T-cellAPC conjugates and inhibits maturation of the immunological synapse.

On the basis of these results,

Gil et al. propose that ligand engagement of TCR-CD3 exposes a PRS in CD3ع that recruits NCK. This is consistent with the observation that the entire cytoplasmic tails of $\mathrm{CD} 3$ subunits have been conserved throughout evolution, and not just the ITAMs. They suggest that full $\mathrm{T}$-cell activation requires a combination of ligand-induced crosslinking, leading to phosphorylation, and ligand-induced conformational change, allowing NCK binding. This is a radical change to our assumptions about TCR signalling. Kirsty Minton

(6)) References and links ORIGINAL RESEARCH PAPER Gil, D. et al Recruitment of Nck by $\mathrm{CD} 3 \varepsilon$ reveals a ligandinduced conformational change essential for T-cell receptor signalling and synapse formation. Cell 109, 901-912 (2002)

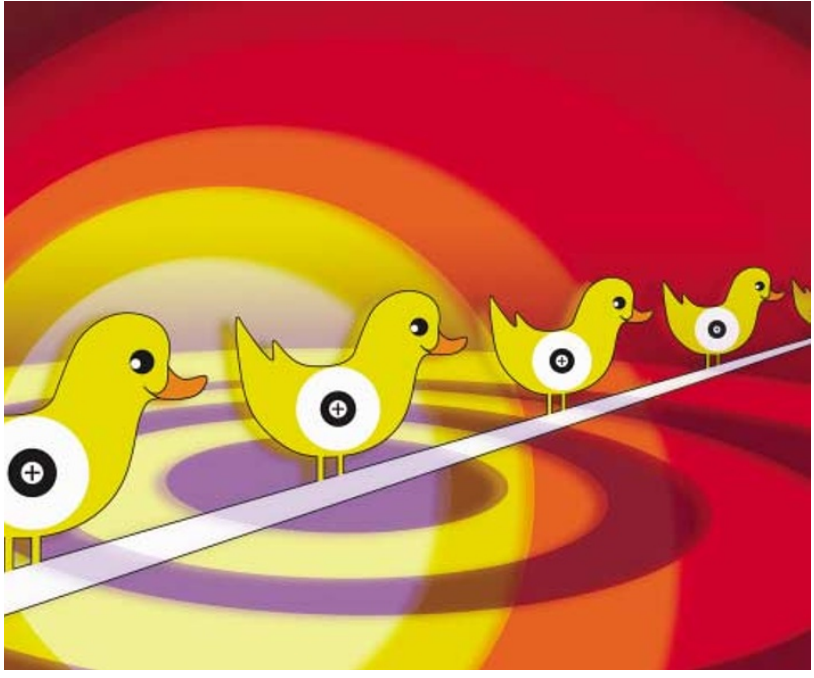

HIV

\section{A new therapeutic target?}

Viruses have evolved various mechanisms to avoid recognition and destruction by the host immune system. By studying these mechanisms, we have learned much about how the immune system functions, and in the process, new therapeutic targets for drug development have been identified. HIV-1 has proven to be a difficult nut to crack in this regard, but a new study in Nature describes the identification of a human protein, CEM15, the function of which is suppressed by viral Vif, leading to the production of infectious virions. This interaction might be a new therapeutic target for drug development.

The Vif-deficient virions that are produced by primary $\mathrm{T}$ cells are non-infectious, such that T cells, and T-cell lines such as CEM, are referred to as non-permissive (NP). By contrast, other cell types, such as CEM-SS (a subclone of the CEM cell line), are termed permissive $(\mathrm{P})$, because they can produce infectious Vif-deficient virions. Cell-fusion experiments have shown that the NP phenotype is dominant over the $\mathrm{P}$ phenotype. These data imply that a factor in the NP cells is able to influence the production of infectious Vif-deficient virions. Malim and colleagues set out to identify this factor by using a PCR-based complementary DNA subtraction strategy to compare CEM (NP) and CEM-SS (P) cells. Subtracted cDNAs were used as probes in northern-blot experiments using RNA from both NP and $P$ cells. The authors identified a cDNA that corresponds to a gene that they have named CEM15, and the transcript was identified in all of the NP cells that were tested. Ectopic expression of CEM15 in a $P$ cell line had no effect on the quantity of Vif-deficient HIV-1 particles produced by these cells, but instead of being infectious, the virus particles were non-infectious.

What is CEM15? Analysis of the CEM15 protein sequence showed that it has marked homology to APOBEC1 (a cytidine deaminase that specifically edits APOB mesenger RNA).

So, CEM15 seems to be responsible for the inability of NP cells to produce infectious Vif-deficient virions, which indicates that CEM15 is the cellular target of Vif. Because of the homology to APOBEC1, the authors speculate that CEM15 might affect Vif-deficient virions by means of interactions with viral RNA. Importantly, the CEM15-Vif interaction might prove to be an important therapeutic target for the development of new drugs to alter virus infectivity. But, the function of CEM15, and how Vif suppresses this function, remain to be determined.

(1) References and links

Elaine Bell

ORIGinAl RESEARCH PAPER Sheey, A. M., Gaddis, N. C., Choi, J. D. \& Malim, M. H. Isolation of a human gene that inhibits HIV-1 infection and is suppressed by the viral Vif protein. Nature July 142002 (DOI 10.1038/nature00939)

FURTHER READING Bell, J. Deamination unlocks diversity. Nature Rev. Immund. July 15 2002 (DO) 10.1038/nri870) | Martin, A. \& Scharff, M. AID and mismatch repair in antibody diversification. Nature Rev. Immunol. 2, 605-614 (2002)

\section{Mapping the maze}

CD1 molecules are MHC class-Ilike glycoproteins that bind lipidbased antigens, but the binding mechanisms that are used by $\mathrm{CD} 1$ molecules to accommodate lipid antigens that seem to be too large for the binding groove have remained unclear. Gadola et al., reporting in Nature Immunology, describe the crystal structures of human CD1B complexed to glycolipid antigens. These new structures reveal that the binding groove is more complex than was previously supposed, which helps to explain the ability of CD1 molecules to bind long lipid chains.

Gadola et al. developed an in vitro system for refolding denatured CD1B molecules in the presence of defined glycolipids. The complexes produced were cystallized and the structures analysed in detail. Compared with MHC class I, the binding groove of CD1B is a complex maze of interconnecting pockets and tunnels - three distinct pockets, named $\mathrm{A}^{\prime}$, $\mathrm{C}^{\prime}$ and $\mathrm{F}^{\prime}$, were defined, as well as a connecting tunnel, termed $\mathrm{T}^{\prime}$. CD1B can bind long lipid chains by accommodating alkyl chains of up to 70 carbons in length in a superchannel formed by the connection of $\mathrm{A}^{\prime}, \mathrm{T}^{\prime}$ and $\mathrm{F}^{\prime}$. The $\mathrm{C}^{\prime}$ pocket can accommodate shorter alkyl chains of $\sim 16$ carbons. An exit portal for the $\mathrm{C}^{\prime}$ pocket, located below the $\alpha 2$ helix, indicates that longer chains might also be accommodated. These structures show how CD1B can adapt to bind lipid ligands of various sizes.

Elaine Bell

6) References and links ORIGINAL RESEARCH PAPER Gadola, S. D. et $\mathrm{al}$. Structure of human $\mathrm{CD} 1 \mathrm{~b}$ with bound ligands at 2.3A, a maze for alkyl chains. Nature Immunol. July 152002 (DOI 10.1038/ni821)

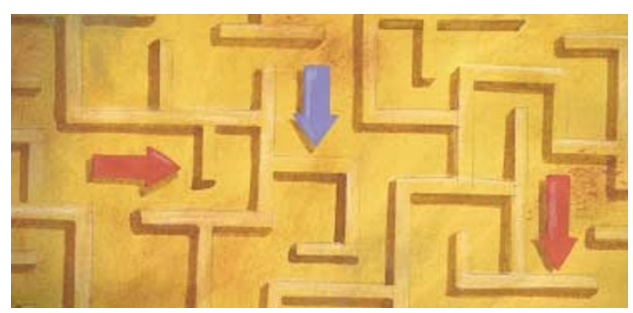

